\title{
A Canadian paediatric brain tumour consortium (CPBTC) phase II molecularly targeted study of imatinib in recurrent and refractory paediatric central nervous system tumours
}

\author{
Sylvain Baruchel ${ }^{a, *}$, Julia R. Sharp ${ }^{a}$, Ute Bartels ${ }^{a}$, Juliette Hukin ${ }^{b}$, Isaac Odame, \\ Carol Portwine $e^{c}$, Doug Strother ${ }^{d}$, Chris Fryer ${ }^{b}$, Jackie Halton, Merrill J. Egorin, \\ Rui Manuel Reis ${ }^{g}$, Olga Martinho ${ }^{g}$, Diana Stempak ${ }^{a}$, Cynthia Hawkins ${ }^{h}$, \\ Janet Gammon ${ }^{a}$, Eric Bouffet ${ }^{a}$
}

\begin{abstract}
${ }^{a}$ New Agent and Innovative Therapy Program, Department of Paediatrics Division of Haematology/Oncology, Hospital for Sick Children, 555 University Avenue, Toronto, Ontario, Canada M5G 1X8

bepartment of Haematology/Oncology, British Columbia Children's Hospital, 4480 Oak Street, Vancouver, British Columbia, Canada V6H 3V4

${ }^{\mathrm{c}}$ Department of Paediatrics, Division of Haematology/Oncology, McMaster University Medical Center, 1200 Main Street West Hamilton, Ontario, Canada L8N $3 Z 5$

${ }^{\mathrm{d} D e p a r t m e n t}$ of Haematology/Oncology, Alberta Children's Hospital, 2888 Shaganappi Trail North West, Calgary, Alberta, Canada T3B 6A8 eDepartment of Haematology/Oncology, Children's Hospital of Eastern Ontario, 401 Smyth Road, Ottawa, Ontario, Canada K1H 8L1

${ }^{f}$ Department of Medicine and Pharmacology, University of Pittsburgh Cancer Institute, 5150 Centre Avenue, 15232 Pittsburgh, PA, USA

gLife and Health Science Research Institute (ICVS), School of Health Sciences, University of Minho, Campus de Gualtar, 4710-057 Braga, Portugal

${ }^{\mathrm{h}}$ Department of Paediatric Laboratory Medicine, Division of Pathology, The Hospital for Sick Children, 555 University Avenue, Toronto, Ontario, Canada M5G 1 X8
\end{abstract}

A R T I C L E I N F O

Article history:

Received 13 February 2009

Received in revised form 16 April

2009

Accepted 7 May 2009

Available online 6 June 2009

Keywords:

Imatinib

Paediatrics

Phase II

Central nervous system neoplasms Brain neoplasms

Platelet-derived growth factor alpha receptor

C-kit

Drug toxicity

Pharmacokinetics

\begin{abstract}
A B S T R A C T
Purpose: To evaluate the safety, efficacy and pharmacokinetics of imatinib in children with recurrent or refractory central nervous system (CNS) tumours expressing KIT and/or PDGFRA.

Methods: Nineteen patients aged 2-18 years, with recurrent or refractory CNS tumours expressing either of the target receptors KIT and/or PDGFRA (by immunohistochemistry) were eligible. Participants received imatinib orally at a dose of $440 \mathrm{mg} / \mathrm{m}^{2} /$ day and toxicities and tumour responses were monitored. Serial blood and cerebrospinal fluid samples for pharmacokinetics were obtained in a subset of consenting patients. Frozen tumour samples were analysed retrospectively for KIT and PDGFRA gene amplification in a subset of patients for whom samples were available.

Results: Common toxicities were lymphopaenia, neutropaenia, leucopaenia, elevated serum transaminases and vomiting. No intratumoural haemorrhages were observed. Although there were no objective responses to imatinib, four patients had long-term stable disease (SD) (38-104 weeks). Our results suggest a possible relationship between KIT expression and maintenance of SD with imatinib treatment; KIT immunopositivity was seen in only $58 \%(11 / 19)$ of study participants overall, but in $100 \%$ of patients with SD at
\end{abstract}

\footnotetext{
* Corresponding author: Tel.: +1 416813 7795; fax: +1 4168135327.

E-mail address: sylvain.baruchel@sickkids.ca (S. Baruchel).

0959-8049/\$ - see front matter @ 2009 Elsevier Ltd. All rights reserved.

doi:10.1016/j.ejca.2009.05.008
} 
38 weeks. All patient tumours showed PDGFRA expression. Pharmacokinetic data showed a high interpatient variability, but corresponded with previously reported values.

Conclusions: Imatinib at $440 \mathrm{mg} / \mathrm{m}^{2} /$ day is relatively safe in children with recurrent CNS tumours, but induced no objective responses. Demonstration of SD in previously progressing patients (KIT-expressing) suggests cytostatic activity of imatinib.

(c) 2009 Elsevier Ltd. All rights reserved.

\section{Introduction}

Central nervous system (CNS) tumours are the second most frequent malignancy and most common form of solid tumour in children. With a 5-year survival rate of $65 \%$, these tumours are the leading cause of cancer-related mortality in children, despite aggressive multi-modal therapy. ${ }^{1}$ In addition, effects of either treatment or the tumour itself often leave survivors of childhood CNS tumours with significant neurologic, neurocognitive and psychosocial sequelae. ${ }^{1,2}$ Better treatment approaches are needed.

Imatinib mesylate (STI571) is a targeted small molecule competitive inhibitor of subclass III receptor tyrosine kinases, including KIT and PDGFRA., ${ }^{3,4}$ KIT is a proto-oncogene that encodes KIT (CD117), ${ }^{5}$ which, when bound to its ligand stem cell factor, is implicated in signalling leading to cell proliferation. ${ }^{6}$ The role of KIT in tumourigenesis has been best studied in gastrointestinal stromal tumours (GISTs) where KIT is commonly mutated, causing constitutive, ligand-independent activations of KIT responsible for oncogenic potential. ${ }^{7,8}$

The two PDGF receptors, $\alpha$ and $\beta$, can be found on fibroblasts, glial cells and vascular smooth muscle cells, where PDGF binding results in cell proliferation, differentiation and migration. ${ }^{9}$ In addition, studies show that imatinib reduces the expression of VEGF, a downstream effector in the PDGF pathway, ${ }^{10}$ suggesting that imatinib may have indirect antiangiogenic activity via its inhibition of PDGFRA. Mutations in both the PDGFRA and KIT genes correlate with GIST tumour response to treatment. ${ }^{4}$

Imatinib treatment has shown significant clinical responses in KIT-expressing GIST ${ }^{11,12}$ in adults, and in chronic myeloid leukaemia (CML) expressing the BCR-ABL fusion protein in both adults ${ }^{13}$ and children. ${ }^{14,15}$ Overexpression of KIT and PDGFRA has been demonstrated in multiple paediatric brain tumours; medulloblastoma, ${ }^{16}$ neuroblastoma, ${ }^{17}$ intracranial germinoma, ${ }^{18}$ and more recently astrocytoma, ${ }^{19}$ showed KIT overexpression, while PDGFRA was overexpressed in paediatric astrocytoma and in a small percentage of paediatric gliomas. ${ }^{19,20}$

Evidence of successful clinical studies in malignancies expressing imatinib targets, and of target expression in various paediatric brain tumours, provided the rationale to conduct a phase II study evaluating efficacy and safety of imatinib in paediatric patients with recurrent or refractory CNS tumours. Objectives of the study were: to assess clinical efficacy, safety and pharmacokinetics of imatinib; to assess the frequency of KIT and PDGFRA expression and to correlate efficacy with expression.

\section{Patients and methods \\ 2.1. Patients}

This was a multi-centre Canadian study. Patients $\leqslant 21$ years of age with histologically confirmed recurrent or refractory CNS tumours expressing KIT and/or PDGFRA by immunohistochemistry were eligible for study. Patients were stratified into those with (stratum 1) or without (stratum 2) leptomeningeal dissemination of disease. At baseline, patients were required to have measurable disease documented by CT or MRI. Leptomeningeal dissemination was defined as positive findings on spine MRI, or as presence of malignant cytology or abnormally high protein in cerebrospinal fluid (CSF).

Additional eligibility criteria included Lansky performance score $\geqslant 40 \%$ or Karnofsky score $\geqslant 50 \%$, life expectancy $\geqslant 8$ weeks, adequate bone marrow function (ANC $\geqslant 1500 / \mu \mathrm{L}$, platelet count $\geqslant 100,000 / \mu \mathrm{L}$ haemoglobin $\geqslant 8.0 \mathrm{~g} / \mathrm{dL}$ ), adequate liver function (total bilirubin $\leqslant 1.5 \times$ upper limit of normal for age, $\mathrm{ALT} \leqslant 2.5 \times$ upper limit of normal for age), adequate renal function (age-adjusted normal serum creatinine or GFR $\geqslant 70 \mathrm{ml} /$ $\mathrm{min} / 1.73 \mathrm{~m}^{2}$ ) and normal coagulation (INR $\leqslant 1.5$, PTT and fibrinogen normal). Negative pregnancy tests were required in postmenarchal females. All patients of reproductive age agreed to effective contraceptive use. Pregnant or breast-feeding women were excluded. Patients requiring drugs known to activate the cytochrome p450 enzyme system (including enzyme-inducing anti-epileptic drugs) and patients with medical conditions that could alter imatinib metabolism were excluded.

The study was approved by the institutional review board of each participating institution and conducted in accordance with the institutional and federal guidelines for human investigations. Patients and parents signed institutional review board-approved informed consent forms before enrolment.

\subsection{Criteria for response and progression}

Tumour response was determined using National Cancer Institute Response Evaluation Criteria in Solid Tumours (RECIST). Progressive disease (PD) was defined as $\geqslant 20 \%$ increase in cross-sectional area of a lesion or the appearance of new lesions or malignant cells in previously normal CSF. Complete response (CR) was defined as the complete disappearance of target lesions, partial response (PR) as at least 30\% decrease in disease measurement, and stable disease (SD) as neither sufficient shrinkage to qualify for PR nor sufficient increase to qualify for PD. Lesions were measured using either CT or MRI (single modality for each patient) at baseline, following course 1 , and following every alternate course thereafter. 


\subsection{Immunostaining}

Immunostaining was centrally performed. About $5 \mu \mathrm{m}$ formalin-fixed, paraffin-embedded tissue sections were mounted on positively charged microscope slides and baked overnight at $60^{\circ} \mathrm{C}$ prior to immunostaining. Immunohistochemistry was performed on the Ventana Benchmark ${ }^{\text {Tu }}$ auto-immuno stainer using the UltraView multimer DAB detection system for the polyclonal rabbit anti-human KIT (CD117) (Dako, Carpinteria, CA, United States of America), and IVIEW DAB, LSAB detection system for the Polyclonal Rabbit anti-human PDGFRA (Santa Cruz Biotechnology, Santa Cruz, CA, USA) at dilutions of 1:50 and 1:100, respectively. Staining was scored on an arbitrary scale based on strength: $0=$ no staining; $1=$ weak staining and $2=$ strong staining. Control tissue cells (duodenal mast cells and angiofibroma cells for KIT and PDGFRA, respectively) were used as an internal scoring guide (score 2).

\subsection{Treatment administration}

Patients had to begin treatment within 14 days of obtaining radiological proof of recurrent or refractory disease. Imatinib was provided by Novartis Pharmaceuticals in $100 \mathrm{mg}$ capsules. Patients in both strata took imatinib orally at a dose of $440 \mathrm{mg} / \mathrm{m}^{2} /$ day (rounded to nearest multiple of $100 \mathrm{mg}$ ) for courses of 28 days with no interruptions. Imatinib was divided BID for doses $\geqslant 600 \mathrm{mg} /$ day, and given to a maximum dose of $800 \mathrm{mg} /$ day. No dose escalation was allowed. Patients with $\mathrm{PD}$ or unacceptable toxicity were taken off study. In the absence of $\mathrm{PD}$, drug-related toxicities were managed by dose interruption or reduction based on the protocol guidelines. Patients who demonstrated SD, PR or CR were eligible to receive therapy for up to 2 years. No other cancer chemotherapy or immunomodulating agents were used.

\subsection{Definition of toxicity}

Toxicities were graded using National Cancer Institute Common Toxicity Criteria (NCI-CTC) Version 2.0. Safety assessments included regular monitoring of haematological parameters, blood chemistry analysis, urinalysis, vital signs, physical condition and weight. For adverse events and serious adverse events, severity, duration, outcome and presumed relationship to study drug were recorded.

\subsection{Pharmacokinetic evaluation}

On day one of treatment, $2 \mathrm{ml}$ of heparinised blood samples were obtained before morning drug administration and at hours 0.5, 1, 2, 3, 4, 8, 11:55 (before second dose if BID dosing), 24 and 48 following drug administration. A trough level on day 8 was obtained prior to drug ingestion. Plasma was prepared by centrifugation and stored at $-80^{\circ} \mathrm{C}$ until it was analysed for drug content. Sampling of CSF was done at baseline in cases of leptomeningeal dissemination, and repeated $4 \mathrm{~h}$ after morning drug administration on day 15, 28 (end of cycle 1) and 56 (end of cycle 2).

Plasma and CSF concentrations of imatinib and its active metabolite N-desmethyl-imatinib (CGP74588) were deter- mined using a validated liquid chromatography/mass spectrometry assay as previously reported..$^{21}$

\subsection{QRT-PCR evaluation for KIT and PDGFRA}

For quantitative real-time PCR (QRT-PCR), $10 \mu \mathrm{m}$ sections of paraffin blocks were cut and selected areas containing at least $85 \%$ of tumour were marked for DNA isolation as previously described. ${ }^{22}$ QRT-PCR was performed with LightCycler (Roche Molecular Biochemicals, Mannheim, Germany). Primers and probes were designed to amplify a 124 bp (exon 18 from PDGFRA gene), $163 \mathrm{bp}$ (exon 17 from KIT gene) and $147 \mathrm{bp}$ (18S gene) specific PCR product, where $18 \mathrm{~S}$ was used as reference gene. PCR amplification conditions, primers and probes for $18 \mathrm{~S}$ and KIT were previously described. ${ }^{22}$ For PDGFRA, conditions were as follows: 5'-TCAGCTACAGATGGCTTGATCC-3' (forward primer), 5'-GCCAAAGTCACAGATCTTCACAAT-3' (reverse primer), 5'-TGTGTCCACCGTGATCTGGCTGC-FL (donator probe), LC640-CGCAACGTCCTCCTGGCACAAGG-3' (acceptor probe).

Evaluation of data was performed using the $\Delta \Delta \mathrm{Ct}$ method: $\Delta \Delta \mathrm{Ct}=\Delta \mathrm{Ct}$ Tumour DNA $-\Delta \mathrm{Ct}$ Normal blood DNA. $\Delta \mathrm{Ct}$ (threshold cycles) is the $\mathrm{Ct}$ of the reference gene minus the $\mathrm{Ct}$ of the target gene. Fold increase of the target gene was calculated by $2(\Delta \Delta \mathrm{Ct})$.Values between 2 and 5 were considered as aneuploidy and values $\geqslant 5$ were considered as gene amplification.

\subsection{Statistical analysis}

The study was designed as an intent-to-treat study. The primary end-point was objective response rate as assessed by RECIST criteria. Secondary end-point criteria included toxicities as assessed by the NCI-CTC, and pharmacokinetic data, including maximum concentration, steady-state concentration and half-life.

\section{Results}

\subsection{Study cohort}

Between August 25, 2003 and March 26, 2007, 19 patients were enroled for the study at 5 study centres: 9 patients without, and 10 patients with, CNS dissemination (strata 1 and 2, respectively). Median age at study entry was 10 (range 3-18) for stratum 1 and 8.5 (range 2-17) for stratum 2. Characteristics of patients are presented in Table 1.

One patient was excluded from analysis of toxicity due to very early progression. Seven patients consented to blood draws for pharmacokinetics but only five were fully evaluable. No patients had 48-hour samples taken on day 1 due to issues of consent. One patient consented to CSF samples for pharmacokinetics analysis. Archival frozen tumour samples for retrospective QRT-PCR analysis of gene amplification were available for three patients.

\subsection{Safety and toxicity}

Safety was assessed in 18 patients who completed $\geqslant 1$ course of imatinib. At the time of study closure, 70 total courses of 


\section{Table 1 - Characteristics of eligible patients at baseline.}

\begin{tabular}{|c|c|c|c|}
\hline & $\begin{array}{c}\text { Stratum } 1(n=9) \\
\text { (No CNS dissemination) }\end{array}$ & $\begin{array}{c}\text { Stratum } 2(n=10) \\
\text { (CNS dissemination present) }\end{array}$ & $\begin{array}{l}\text { All patients } \\
(n=19)\end{array}$ \\
\hline \multicolumn{4}{|l|}{ Age at enrolment (years) } \\
\hline Median (range) & $10(3-18)$ & $8.5(2-17)$ & $9(2-18)$ \\
\hline \multicolumn{4}{|l|}{ Gender } \\
\hline Female & 3 & 4 & 7 \\
\hline Male & 6 & 6 & 12 \\
\hline \multicolumn{4}{|l|}{ Histology } \\
\hline Astrocytoma & 2 & 1 & 3 \\
\hline Ependymoma & 3 & 2 & 5 \\
\hline GBM & 1 & 1 & 2 \\
\hline Medulloblastoma/PNET & 2 & 6 & 8 \\
\hline Neurocytoma & 1 & 0 & 1 \\
\hline \multicolumn{4}{|l|}{ Receptor staining } \\
\hline PDGFRA + (\%) & $9(100 \%)$ & $10(100 \%)$ & $19(100 \%)$ \\
\hline $\mathrm{KIT}+(\%)$ & $7(77.8 \%)$ & $4(40 \%)$ & $11(57.9 \%)$ \\
\hline \multicolumn{4}{|l|}{ Performance status ${ }^{\mathrm{a}}$} \\
\hline Median (range) & $70(60-100)$ & $90(50-100)$ & $80(50-100)$ \\
\hline \multicolumn{4}{|l|}{ Weeks since diagnosis } \\
\hline Median (range) & $9(4-43)$ & $13(2-39)$ & $12(2-43)$ \\
\hline \multicolumn{4}{|c|}{ Disease status prior to study entry } \\
\hline $\mathrm{SD}$ & 2 & 1 & 3 \\
\hline $\mathrm{PD}$ & 7 & 9 & 16 \\
\hline \multicolumn{4}{|l|}{ Relapse number ${ }^{\mathrm{b}}$} \\
\hline Median (range) & $2(1-3)$ & $2(1-5)$ & $2(1-5)$ \\
\hline \multicolumn{4}{|l|}{ Prior chemotherapy regimens } \\
\hline Median (range) & $1(1-4)$ & $2(0-3)$ & $2(0-4)$ \\
\hline 0 & 0 & 1 & 1 \\
\hline 1 & 6 & 2 & 8 \\
\hline 2 & 2 & 4 & 6 \\
\hline 3 & 0 & 3 & 3 \\
\hline 4 & 1 & 0 & 1 \\
\hline \multicolumn{4}{|l|}{ Prior RT regimens } \\
\hline Median (range) & $1(0-2)$ & $1(1-1)$ & $1(0-2)$ \\
\hline 0 & 1 & 0 & 1 \\
\hline 1 & 7 & 10 & 17 \\
\hline 2 & 1 & 0 & 1 \\
\hline \multicolumn{4}{|l|}{ Prior BMT } \\
\hline Number of patients & 0 & 1 & 1 \\
\hline
\end{tabular}

imatinib had been administered: 57 in stratum 1, and 13 in stratum 2. Median number of cycles per patient was one for stratum 1 (range 1-26), and one for stratum 2 (range 1-4). Most patients received imatinib daily with no interruptions. However, two patients had $\geqslant 7$ days of treatment interruption due to toxicities likely related to imatinib: one patient had grade 3 diarrhoea in courses 2 and 4, with treatment interruption for 7 and 8 days, respectively; another patient had hypoalbuminaemia and oedema in cycle 7, with treatment interruption for 20 consecutive days. Both the patients were in stratum 1. Dose reductions following adverse events were made in two stratum 1 patients after course 1 . Of all study pa- tients, five (four in stratum 1) received imatinib for $\geqslant 16$ weeks, and four (all stratum 1 ) received for $\geqslant 38$ weeks.

Course 1 adverse events are summarised in Table 2. Haematological toxicities were common during course 1; lymphopaenia, neutropaenia, thrombocytopaenia and leucopaenia all occurred in $\geqslant 4(20 \%)$ of patients. The most commonly reported non-haematological toxicities in course 1 were vomiting, nausea, diarrhoea, elevated ALT, skin rash and fatigue.

Three serious adverse events were considered related to imatinib. One patient was hospitalised during course 1 for disseminated shingles infection with high-grade thrombocytopaenia and ALT/AST elevation; this patient died while on 
Table 2 - Grades 1-2 and 3-4 NCI-CTC imatinib-related toxicities reported during course $1(n=18)$.

\begin{tabular}{|c|c|c|c|c|}
\hline \multirow[t]{2}{*}{ Event } & \multicolumn{2}{|c|}{ Grades 1-2 } & \multicolumn{2}{|c|}{ Grades 3-4 } \\
\hline & No. pts & $(\%)$ & No. pts & $(\%)$ \\
\hline \multicolumn{5}{|l|}{ Haematological } \\
\hline Lymphopaenia & 7 & 38.9 & 1 & 5.6 \\
\hline Neutropaenia & 4 & 22.2 & $2^{b}$ & 11.1 \\
\hline Thrombocytopaenia & 4 & 22.2 & 0 & 0 \\
\hline Leukocytes & 6 & 33.3 & 1 & 5.6 \\
\hline \multicolumn{5}{|l|}{ Non-haematological } \\
\hline ALT & 5 & 27.8 & 1 & 5.6 \\
\hline Diarrhoea & 3 & 16.7 & 0 & 0 \\
\hline Vomiting & 6 & 33.3 & 1 & 5.6 \\
\hline Pulmonary (wheeze) & & 0 & 1 & 5.6 \\
\hline Infection & 1 & 5.6 & 1 & 5.6 \\
\hline Fatigue & 2 & 11.1 & 1 & 5.6 \\
\hline Creatinine & 1 & 16.7 & 0 & 0 \\
\hline Constipation & 1 & 5.6 & 0 & 0 \\
\hline Dehydration & 1 & 5.6 & 0 & 0 \\
\hline Weight loss & 1 & 5.6 & 0 & 0 \\
\hline Nausea & 4 & 22.2 & 0 & 0 \\
\hline Headache & 2 & 11.1 & 0 & 0 \\
\hline Blurred vision & 1 & 5.6 & 0 & 0 \\
\hline Skin rash & 3 & 16.7 & 0 & 0 \\
\hline Cramping/spasm & 1 & 5.6 & 0 & 0 \\
\hline Swelling & 1 & 5.6 & 0 & 0 \\
\hline Dizziness & 1 & 5.6 & 0 & 0 \\
\hline Ataxia & 1 & 5.6 & 0 & 0 \\
\hline Poor appetite & 1 & 5.6 & 0 & 0 \\
\hline Haematuria & 1 & 5.6 & 0 & 0 \\
\hline
\end{tabular}

study due to PD. Another patient experienced severe hypoalbuminaemia and oedema, vomiting, diarrhoea and constipation post-course 7. After recovery, treatment was resumed at $75 \%$ dose. Another patient was hospitalised during course 9 for pneumonia, thrombocytopaenia, diarrhoea, ascites and haemorrhagic pleural and pericardial effusions, and was taken off study due to unacceptable toxicity. Five patients died within 28 days of the last dose of imatinib. None were related to the study drug.

\subsection{Outcome results}

Tumour response was assessed in all 19 patients. There were no PRs or CRs to treatment. Four patients maintained SD $\geqslant 38$ weeks on treatment (all stratum 1), including one patient each with ependymoma, astrocytoma, medulloblastoma and primitive neuroectodermal tumour (PNET). All others had progressed by 16 weeks. The PNET patient had SD with a refractory tumour upon study entry, maintained SD for 104 weeks ( 26 courses), and was taken off study after completion of 2 years. This patient progressed 6 months later. Of the patients who maintained SD, three of four were progressing prior to study entry. The PNET patient entered the study with refractory SD, maintained SD for 104 weeks (26 courses) with imatinib treatment, and was taken off study after completion of 2 years. This patient progressed 6 months later. The astrocytoma, medulloblastoma, and ependymoma patients all had PD upon study entry and maintained SD for 38, 38, and 39 weeks, respectively, with imatinib treatment. The medullo- blastoma patient still had SD when taken off study for unacceptable toxicity (pericardial and pleural effusion) at 38 weeks. The others were taken off study for PD.

\subsection{Pharmacokinetic analyses}

Pharmacokinetic data were available for five patients. Based on body surface area, one patient received $500 \mathrm{mg} /$ day, three received $600 \mathrm{mg} /$ day (300 mg BID), and one received $700 \mathrm{mg} /$ day (300 and $400 \mathrm{mg}$ ). Patients receiving doses of BID underwent PK analysis based on the $300 \mathrm{mg}$ morning dose. CSF samples were available for one patient at the $300 \mathrm{mg}$ BID dose level. Considerable interpatient variability of imatinib pharmacokinetics was observed. Details are reported in Table 3.

The CSF samples were obtained at pre-treatment, day 15 and day 28. At day 28 the sample was contaminated with blood and was not useable. At day 15, imatinib concentration was $92 \mathrm{ng} / \mathrm{ml}$ and CGP74588 concentration was $13 \mathrm{ng} / \mathrm{ml}$. This was less than $5 \%$ of corresponding plasma levels, suggesting a weak penetration of imatinib into the CNS.

\subsection{Immunostaining results}

While assessing study eligibility, 35 total patient tumour samples were tested for KIT and PDGFRA immunostaining. All 35 patients were immunopositive for PDGFRA, while only 17 (49\%) were immunopositive for KIT. No single tumour type showed a reliable pattern of KIT expression. Only 5 of 8 (63\%) medulloblastoma/PNET patients tested positive for 
Table 3 - Pharmacokinetic data based on serum concentration after morning dose.

\begin{tabular}{|c|c|c|c|}
\hline \multicolumn{4}{|c|}{$\begin{array}{ll}\text { Dose level } & \text { Dos }\end{array}$} \\
\hline & \multicolumn{2}{|l|}{$300 \mathrm{mg}$} & \multirow[t]{2}{*}{$500 \mathrm{mg}$} \\
\hline & Median (ng/ml) & Range (ng/ml) & \\
\hline Day 1 & $(n=4)$ & $(n=1)$ & \\
\hline Imatinib $C_{\max }$ & 2549 & $1728-2968$ & 4867 \\
\hline Imatinib trough & 277 & $123-350$ & 862 \\
\hline CGP74588 $C_{\max }$ & 345 & $170-407$ & 1060 \\
\hline CGP74588 trough & 123 & $65-350$ & 322 \\
\hline Day 8 & $(n=3)$ & $(n=1)$ & \\
\hline Imatinib concentration & 3297 & $2103-3725$ & 2067 \\
\hline CGP74588 concentration & 570 & $469-897$ & 819 \\
\hline
\end{tabular}

KIT, which is inconsistent with our previous findings that 10 of 10 medulloblastoma samples expressed KIT. ${ }^{16}$

Of the 19 study patients, 11 (58\%) were positive for KIT. Of note, however, is that of the 4 patients with SD at 38 weeks, all $4(100 \%)$ were KIT positive.

\subsection{QRT-PCR evaluation for KIT and PDGFRA}

Archival pre-treatment tumour pathology specimens were available for three of 19 study patients. The samples were evaluated retrospectively for amplification of KIT and PDGFRA genes to assess for correlation with response to imatinib. None of the tumours samples exhibited gene amplification of either KIT or PDGFRA, thus we were unable to assess relation to outcome.

\section{Discussion}

Imatinib at a dose of $440 \mathrm{mg} / \mathrm{m}^{2} /$ day was evaluated in the treatment of recurrent or refractory paediatric CNS tumours expressing KIT or PDGRFA. Given the efficacy of imatinib treatment in cancers expressing these targets and confirmation of expression in patients' tumour samples, there was strong rationale to evaluate imatinib in this setting.

No objective tumour responses (either CR or PR) were observed. Another recent paediatric study of imatinib treatment for CNS tumours shows similarly disappointing results, with no CRs observed, and very low PR rates. ${ }^{23} \mathrm{~A}$ recent study in adult recurrent gliomas showed similarly poor clinical responses. $^{24}$

In this study, four patients $(21 \%)$ maintained SD longer than 38 weeks, yet all but one had progressed by 1 year. This is congruent with the above-mentioned study in adult recurrent gliomas, in which imatinib treatment resulted in SD in $25 \%$, and in PR in only $5 \%$ of patients. ${ }^{24}$ Another recent study on recurrent adult gliomas showed SD in $20 \%$, and PR in $4 \%$ of patients. ${ }^{25}$ These data suggest that imatinib may have cytostatic activity in adult CNS tumours. Given that some patients with PD just prior to study entry achieved SD with imatinib treatment, our data suggest some cytostatic activity of imatinib in paediatric CNS tumours. Given that all four patients with SD at 38 weeks had KIT-expressing tumours, KIT may correlate with maintenance of SD with imatinib treatment. However, we cannot draw firm conclusions about cytostatic activity of imatinib or the correlation of KIT with SD, because the heterogeneity and rarity of these tumours limit the availability of historical controls for comparison.

There are several potential explanations for lack of objective response to imatinib treatment. Imatinib penetration of the blood brain barrier (BBB) is poor. Recent reports have documented CSF imatinib levels 40- to 92-fold lower than the corresponding plasma levels in adults with intact BBBs treated for CML and acute lymphoblastic leukaemia. ${ }^{26,27}$ Data from our study showed that CSF levels of imatinib are less than $5 \%$ of the corresponding plasma levels. Access to the CSF is likely limited by drug efflux pumps such as P-glycoprotein, which is highly expressed in the $\mathrm{BBB} .^{28}$ Low CSF concentrations of imatinib likely contribute to its limited efficacy in CNS tumours, despite the possibility for partial BBB disruption in these patients. Dasatinib, a second-generation receptor tyrosine kinase inhibitor crosses the BBB effectively despite P-glycoprotein expression, and has shown a significant clinical activity in adult CML patients with CNS involvement. ${ }^{29}$ Future assessment of the efficacy of secondgeneration tyrosine kinase inhibitors in CNS tumours should be considered.

Previous studies assessing imatinib in CNS tumours, which have based treatment rationale on published findings that these tumour types frequently overexpress imatinib targets, have found similarly limited evidence of clinical efficacy of imatinib as a single agent. To our knowledge, this study is the first to use target receptor expression status as eligibility criteria for an imatinib study. Despite the fact that the patient tumours had confirmed the expression of imatinib targets, treatment showed limited clinical activity in paediatric CNS tumours. This suggests that target expression is not sufficient to predict imatinib activity. However, the mutation status of both KIT and PDGFRA has been found retrospectively to be predictive of imatinib response in GIST. ${ }^{4,30}$ In addition, the specific mutation genotype of KIT is predictive of the imatinib dose required for clinical responses in GIST; tumours with exon 11 mutations responded to low dose imatinib, while 
tumours with exon 9 mutations or wild-type KIT benefited from treatment with higher doses of imatinib. ${ }^{31}$ Given these findings, future studies of imatinib combinations in paediatric CNS tumours should consider mutational analysis, rather than expression, of KIT and PDGFRA as potential predictors of tumour response to imatinib.

This study confirmed the phase II dose of $440 \mathrm{mg} / \mathrm{m}^{2} /$ day for imatinib treatment in children. ${ }^{14}$ Toxicities were acceptable and all had been previously reported in children. ${ }^{14,23}$ None of the patients experienced intratumoural haemorrhage, a previously reported toxicity associated with imatinib treatment for solid tumours in children and adults. ${ }^{23,25}$

Pharmacokinetic analysis showed a considerable interpatient variability, as seen in previously published paediatric studies using a similar dosing. ${ }^{14,23}$ Compared with these studies, our data show similar ranges for serum $C_{\max }$ on day 1 and day 8 for both imatinib and its active metabolite CGP74588.

The findings of SD in this study and prior adult studies suggest cytostatic activity of imatinib in some CNS tumours. ${ }^{24,25}$ In a recent phase I study of recurrent malignant gliomas in adults, combination of imatinib with temozolomide was well tolerated and showed promising results, with $12 \%$ of patients achieving PR or CR. ${ }^{32}$ Imatinib in combination with a cytotoxic agent may have a similar clinical efficacy in paediatric CNS tumours. Future preclinical and phase I studies are needed to evaluate the safety and preliminary evidence of efficacy of this treatment.

\section{Role of the funding sources}

The study was supported by Novartis and sponsored by the principal investigator. Data analysis and manuscript writing were performed by the principal investigator and second author. Decision to submit to EJC was made by the principal investigator.

\section{Conflict of interest statement}

None declared.

\section{Acknowledgment}

Imatinib mesylate provided by Novartis Canada.

\section{R E F E R E N C E S}

1. Gurney JG, Smith MA, Bunin GR. CNS and miscellaneous intracranial and intraspinal neoplasms. In: Ries LAG, Smith MA, Gurney JG, et al., editors. Cancer incidence and survival among children and adolescents: United States SEER program 1975-1995, NIH Pub. No. 99-4649. Bethesda, MD: National Cancer Institute, SEER Program; 1999. p. 51-63.

2. Radcliffe J, Bunin GR, Sutton LN, Goldwein JW, Phillips PC. Cognitive deficits in long-term survivors of childhood medulloblastoma and other noncortical tumors: agedependent effects of whole brain radiation. Int J Deu Neurosci 1994;12(4):327-34.
3. Buchdunger E, Cioffi CL, Law N, et al. Abl protein-tyrosine kinase inhibitor STI571 inhibits in vitro signal transduction mediated by c-kit and platelet-derive $d$ growth factor receptors. J Pharmacol Exp Ther 2000;295:139-45.

4. Heinrich MC, Griffith DJ, Druker BJ, Wait CL, Ott KA, Zigler AJ. Inhibition of c-kit receptor tyrosine kinase by STI571, selective tyrosine kinase inhibitor. Blood 2000;965:925-32.

5. Williams DE, Eisenman J, Baird A, et al. Identification of a ligand for the c-kit proto-oncogene. Cell 1990;63(1):167-74.

6. Geissler EN, Ryan MA, Housman DE. The dominant-white spotting (W) locus of the mouse encodes the c-kit protooncogene. Cell 1988;55:185-92.

7. Hirota S, Isozaki K, Moriyama Y, et al. Gain-of-function mutations of c-kit in human gastrointestinal stromal tumors. Science 1998;279:577-80.

8. Isozaki K, Terris B, Belghiti J, Schiffmann S, Hirota S, Vanderwinden JM. Germline-activating mutation in the kinase domain of KIT gene in familial gastrointestinal stromal tumors. Am J Pathol 2000;157:1581-5.

9. Williams LT. Signal transduction by the platelet-derived growth factor receptor. Science 1989;243(4898):1564-70 [Review].

10. Beppu K, Jaboine J, Merchant MS, Mackall CL, Thiele CJ. Effect of imatinib mesylate on neuroblastoma tumorigenesis and vascular endothelial growth factor expression. J Natl Cancer Inst 2004;96(1):46-55.

11. van Oosterom AT, Judson IR, Verweij J, et al. Update of phase I study of imatinib (STI571) in advanced soft tissue sarcomas and gastrointestinal stromal tumors: a report of the EORTC Soft Tissue and Bone Sarcoma Group. Eur J Cancer 2002;38(5):S83-7.

12. Demetri GD, von Mehren M, Blanke CD, et al. Efficacy and safety of imatinib mesylate in advanced gastrointestinal stromal tumors. N Engl J Med 2002;347(7):472-80.

13. Talpaz M, Silver RT, Druker BJ, et al. Imatinib induces durable hematologic and cytogenetic responses in patients with accelerated phase chronic myeloid leukemia: results of a phase 2 study. Blood 2002;99(6):1928-37.

14. Champagne MA, Capdeville R, Krailo M, et al. Imatinib mesylate (STI571) for treatment of children with Philadelphia chromosome-positive leukemia: results from a Children's Oncology Group phase 1 study. Blood 2004;104(9):2655-60.

15. Kolb EA, Pan Q, Ladanyi M, Steinherz PG. Imatinib mesylate in Philadelphia chromosome-positive leukemia of childhood. Cancer 2003;98(12):2643-50.

16. Chilton-Macneill S, Ho M, Hawkins C, Gassas A, Zielenska M, Baruchel S. C-kit expression and mutational analysis in medulloblastoma. Pediatr Deu Pathol 2004;7(5):493-8.

17. Smithey BE, Pappo AS, Hill DA. C-kit expression in pediatric solid tumors: a comparative immunohistochemical study. Am J Surg Pathol 2002;26(4):486-92.

18. Sakuma Y, Sakurai S, Oguni S, Satoh M, Hironaka M, Saito K. c-Kit gene mutations in intracranial germinomas. Cancer Sci 2004;95(9):716-20.

19. Liang ML, Ma J, Ho M, et al. Tyrosine kinase expression in pediatric high grade astrocytoma. J Neurooncol 2008;87(3):247-53.

20. Di Sapio A, Morra I, Pradotto L, Guido M, Schiffer D, Mauro A. Molecular genetic changes in a series of neuroepithelial tumors of childhood. J Neurooncol 2002;59(2):117-22.

21. Parise RA, Ramanathan RK, Hayes MJ, Egorin MJ. Liquid chromatographic-mass spectrometric assay for quantitation of imatinib and its main metabolite (CGP 74588) in plasma. J Chromatogr B Analyt Technol Biomed Life Sci 2003;791(1-2): 39-44.

22. Gomes AL, Reis-Filho JS, Lopes JM, et al. Molecular alterations of KIT oncogene in gliomas. Cell Oncol 2007;29:399-408.

23. Pollack IF, Jakacki RI, Blaney SM, et al. Phase I trial of imatinib in children with newly diagnosed brainstem and recurrent 
malignant gliomas: a pediatric brain tumor consortium report. Neuro Oncol 2007;9(2):145-60.

24. Raymond E, Brandes AA, Dittrich C, et al. European Organisation for Research and Treatment of Cancer Brain Tumor Group Study. Phase II study of imatinib in patients with recurrent gliomas of various histologies: a European Organisation for Research and Treatment of Cancer Brain Tumor Group Study. J Clin Oncol 2008;26(28):4659-65.

25. Wen PY, Yung WK, Lamborn KR, et al. Phase I/II study of imatinib mesylate for recurrent malignant gliomas: North American brain tumor consortium study 99-08. Clin Cancer Res 2006;12(16):4899-907.

26. Takayama N, Sato N, O’Brien SG, Ikeda Y, Okamoto S. Imatinib mesylate has limited activity against the central nervous system involvement of Philadelphia chromosome-positive acute lymphoblastic leukaemia due to poor penetration into cerebrospinal fluid. Br J Haematol 2002;119(1):106-8.

27. Bornhauser M, Jenke A, Freiberg-Richter J, et al. CNS blast crisis of chronic myelogenous leukemia in a patient with a major cytogenetic response in bone marrow associated with low levels of imatinib mesylate and its $\mathrm{N}$-desmethylated metabolite in cerebral spinal fluid. Ann Hematol 2004;83(6):401-2.
28. Dai H, Marbach P, Lemaire M, Hayes M, Elmquist WF. Distribution of STI-571 to the brain is limited by Pglycoprotein-mediated efflux. J Pharmacol Exp Ther 2003;304(3):1085-92.

29. Porkka K, Koskenvesa P, Lundán T, et al. Dasatinib crosses the blood-brain barrier and is an efficient therapy for central nervous system Philadelphia chromosome-positive leukemia. Blood 2008;112(4):1005-12.

30. Debiec-Rychter M, Dumez H, Judson I, et al. EORTC Soft Tissue and Bone Sarcoma Group. Use of c-KIT/PDGFR mutational analysis to predict the clinical response to imatinib in patients with advanced gastrointestinal stromal tumours entered on phase I and II studies of the EORTC Soft Tissue and Bone Sarcoma Group. Eur J Cancer 2004;40(5):689-95.

31. Debiec-Rychter M, Sciotb R, Le Cesned A, et al. KIT mutations and dose selection for imatinib in patients with advanced gastrointestinal stromal tumours. Eur J Cancer 2006;42:1093-103.

32. Reardon DA, Desjardins A, Vredenburgh JJ, et al. Safety and pharmacokinetics of dose-intensive imatinib mesylate plus temozolomide: phase 1 trial in adults with malignant glioma. Neuro Oncol 2008;10(3):330-40. 